\title{
The investigation of valid criteria for hospitalization and discharge in patients with limb cellulitis: a prospective cohort study
}

\section{Samaneh Abiri', Mahdi Foroughian², Hamideh Akbar ${ }^{3}$, Neema John Mehramiz ${ }^{4}$, Naser Hatami ${ }^{5}$, Abdol Ali Ameri $^{5}$, Navid Kalani ${ }^{6,7}$, Esmaeil Rayat Dost ${ }^{1 *}$, Saeed Barazandehpour ${ }^{8}$}

\author{
'Department of Emergency Medicine, Jahrom University of Medical Sciences, Jahrom, Iran \\ 2Department of Emergency Medicine, Faculty of Medicine, Mashhad University of Medical Sciences, Mashhad, Iran \\ ${ }^{3}$ Department of Emergency Medicine, Tehran University of Medical Sciences, Tehran, Iran \\ ${ }^{4}$ Department of Psychiatry Neurology, Banner University Medical Center, Tucson, AZ, USA \\ ${ }^{5}$ Student Research Committee, Jahrom University of Medical Sciences, Jahrom, Iran \\ ${ }^{6}$ Anesthesiology, Critical Care, and Pain Management Research Center, Jahrom University of Medical Sciences, Jahrom, Iran \\ ${ }^{7}$ Research center for social Determinants of Health, Jahrom University of Medical Sciences, Jahrom, Iran \\ ${ }^{8}$ Department of Emergency Medicine, Kerman University of Medical Sciences, Kerman, Iran
}

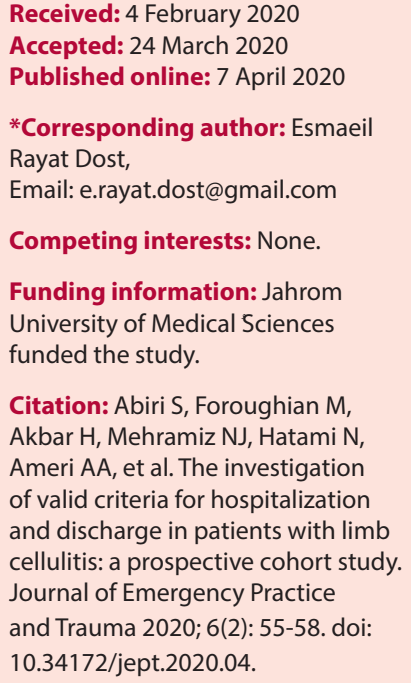

Citation: Abiri S, Foroughian $\mathrm{M}$ Akbar H, Mehramiz NJ, Hatami N, Ameri $A A$, et al. The investigation of valid criteria for hospitalization and discharge in patients with limb cellulitis: a prospective cohort study. Journal of Emergency Practice and Trauma 2020; 6(2): 55-58. doi: 10.34172/jept.2020.04

\begin{abstract}
Objective: The purpose of this study was to evaluate a valid model for patients' admission or discharge from emergency services to improve the health system and reduce costs.

Methods: This study was carried out using a prospective cohort method. The study population was patients with limb cellulitis referring to the emergency department of Peymanieh hospital. In this research, the study participants were separated into two groups based on the duration of hospitalization (hospital stay less than 24 hours or longer than 24 hours), then the patients were again separated into 4 groups based on the classification of the the Clinical Resource Efficiency Support Team (CREST) guideline, which in each of these groups the mean age, gender, and the prevalence of underlying diseases were identified and the final outcome for each group was determined after one week from the visit to the hospital.

Results: Peripheral vascular disease, history of injection drug use, immunodeficiency and congenital immune deficiency had a significant relationship with the rate of hospitalization and recurrence. There was a significant relationship between class 1 disease and hospitalization for less than 24 hours, classes 2 and 3, and hospitalization for more than 24 hours $(P<0.001)$. There was a significant relationship between grade 1 disease and non-recourse, grade 3 and recurrence within one week after initiation of the treatment $(P<0.001)$. But there was no relationship between grade 2 and grade 4 and the referral of the patient after treatment. Conclusion: Corset Scale is a reliable scale for assessing the severity of the disease to determine the process of cellulite treatment for outpatient or hospitalization.

Keywords: Crest Scale, Cellulitis, Limb, Infection
\end{abstract}

\section{Introduction}

Cellulitis is a prevalent and potentially serious skin bacterial infection (1). Cellulitis appears as a red, swollen area on the skin that is warm and sensitive and may spread rapidly (2). It can occur on the entire body's skin and even facial skin. Cellulite may affect only the top layer of the skin, but in some cases, the infection may reach the subcutaneous tissues and from there it may spread to the lymph nodes and bloodstream (3). Fever, tachycardia, hypothermia, hypotension and confusion may also be manifestations of systemic toxicity (4). Cellulite is treated by outpatient or hospitalization care (5). Some patients with cellulitis require multiple hospitalizations due to the recurrence of the disease. Cellulite is known to be related to reduced mortality; however, it can affect a large number of elderly and disabled patients (6). Besides, reports of aggressive group A streptococcal infections associated with shock and untreated wounds have increased in recent years, reminding the importance of the disease (7). Hospitalization of patients rather than outpatient care has been of interest to physicians in improving patient satisfaction, as the cost of medical care for patients has increased (8). Therefore, the classification of patients with cellulitis to determine the risks and complications 
can help physicians make the decisions that are most appropriate for care, such as outpatient or hospitalization (9). This research was designed to evaluate and develop a valid model for hospital admission or discharge from the emergency service to improve the health system and reduce costs.

\section{Methods}

This was a prospective cohort study. The population investigated in this study was patients with limb cellulitis referred to the emergency department of Peymanieh hospital, Jahrom, Iran. Informed consent of all individuals was granted and the information of the study participants was kept confidential by the researcher. Cellulite caused by any external factors such as bleeding ulcer, diabetic foot ulcer, and surgical complications were excluded from the study. Patients were divided into two groups according to the length of hospitalization (duration of hospitalization less than 24 hours and hospitalization more than 24 hours). Then patients were classified into 4 categories based on the Clinical Resource Efficiency Support Team (CREST) guidelines on the management of cellulitis in adults. A checklist which was confirmed by 3 experts and consisted of demographic variables (age, sex, and underlying disease) as well as treatment outcomes was used. The treatment outcome was the duration of hospitalization (less than 24 hours or more than that). The final outcome was considered as the return or not the return of patients 1 week after discharge from the hospital due to treatment failure. Diabetes, peripheral vascular disease, history of using injectable ingredients, history of bite, and immune deficiency history were evaluated. The data were analyzed by descriptive (mean and standard deviation) and analytical ( $t$ test, chi-square, and regression) tests using SPSS software version 19.

\section{Results}

In the present study, 100 subjects participated. 29 subjects were under 30 years (29\%), 16 (16\%) were 30 to 35 years, $14(14 \%)$ were 35 to 40 years and $41(41 \%)$ were over 40 years. Fifty patients (50\%) were males and 50 (50\%) were females. Chi-square test comparing the gender distribution of samples across different age groups showed that there was no difference in gender between different age groups $(P=0.758)$. As shown in Table 1 , there was no significant difference in the time of hospitalization between diabetic and non-diabetic patients or patients having or not having a history of bite $(P>0.05)$. But peripheral vascular disease, history of using injectable ingredients, congenital immune deficiency and immune deficiency history were associated with more than 24 -hour hospitalization $(P<0.05)$. Also, there was no significant difference in the treatment failure (return 1 week after discharge) between diabetic and nondiabetic patients or patients having or not having a history of bite $(P>0.05)$. Conversely, peripheral vascular disease, history of using injectable ingredients, congenital immune deficiency and immune deficiency history were associated with treatment failure $(P<0.05)$.

According to the CREST criteria, 52 patients (52\%) were in class $1,28(28 \%)$ in class $2,18(18 \%)$ in class 3 , and $2(2 \%)$ were classified in class 4 (as depicted in Table 2). Based on the chi-square test, there was a significant difference between the CREST scale and the time of hospitalization $(P=0.001)$. There was a significant relationship between class 1 cellulitis and hospitalization for less than 24 hours and a significant relation between class 2 and 3 and

Table 1. Studied variables based on hospitalization time and patient return to the hospital within one week after discharge

\begin{tabular}{|c|c|c|c|c|c|c|}
\hline \multirow[t]{2}{*}{ Variables } & \multicolumn{2}{|c|}{ Hospitalization (n) } & \multirow[t]{2}{*}{$P$ value } & \multicolumn{2}{|c|}{$\begin{array}{c}\text { Patient return to the hospital within } 1 \\
\text { week after discharge (n) }\end{array}$} & \multirow[t]{2}{*}{$P$ value } \\
\hline & $<24 \mathrm{~h}$ & $>24 h$ & & Yes & No & \\
\hline Diabetes & 1 & 7 & 0.166 & 1 & 7 & 0.914 \\
\hline Peripheral vascular disease & 1 & 22 & 0.023 & 15 & 8 & 0.001 \\
\hline History of using injectable ingredients & 4 & 7 & 0.001 & 3 & 6 & 0.001 \\
\hline Bite history & 2 & 12 & 0.303 & 3 & 8 & 0.063 \\
\hline Congenital immune deficiency & 0 & 5 & 0.003 & 4 & 1 & 0.001 \\
\hline Immune deficiency history & 1 & 7 & 0.019 & 7 & 1 & 0.009 \\
\hline
\end{tabular}

Table 2. Evaluated CREST criteria of patients based on hospitalization time and patient return to the hospital within one week after discharge

\begin{tabular}{|c|c|c|c|c|c|c|}
\hline \multirow{2}{*}{ CREST criteria } & \multicolumn{2}{|c|}{ Hospitalization (n) } & \multirow{2}{*}{$P$ value } & \multicolumn{2}{|c|}{$\begin{array}{c}\text { Patient return to the hospital within } 1 \\
\text { week after discharge (n) }\end{array}$} & \multirow{2}{*}{$P$ value } \\
\hline & $<24 \mathrm{~h}$ & $>24 \mathrm{~h}$ & & Yes & No & \\
\hline Class I & 49 & 3 & \multirow{4}{*}{0.001} & 1 & 51 & \multirow{4}{*}{0.001} \\
\hline Class II & 1 & 27 & & 10 & 18 & \\
\hline Class III & 1 & 17 & & 15 & 3 & \\
\hline Class IV & 0 & 2 & & 2 & 0 & \\
\hline
\end{tabular}


Table 3. Vital sign of patients based on hospitalization time

\begin{tabular}{|c|c|c|c|}
\hline \multirow{2}{*}{ Vital sign variables } & \multicolumn{2}{|c|}{ Hospitalization (n) } & \multirow{2}{*}{$P$ value } \\
\hline & $<24 h$ & $>24 h$ & \\
\hline Temperature & $36.8 \pm 0.35$ & $37.64 \pm 0.77$ & 0.001 \\
\hline Respiratory rate & $14 \pm 3.36$ & $17.41 \pm 4.24$ & 0.001 \\
\hline Heart rate & $77.66 \pm 7.44$ & $84.59 \pm 6.65$ & 0.001 \\
\hline Systolic blood pressure & $119.68 \pm 9.46$ & $128.86 \pm 11.3$ & 0.001 \\
\hline Diastolic blood pressure & $119.68 \pm 9.46$ & $128.86 \pm 11.3$ & 0.001 \\
\hline
\end{tabular}

hospitalization for more than 24 hours $(P<0.001)$.

Finally, our analysis showed a significant relationship between the length of hospitalization and the likelihood of recurrence during the week after discharge $(P=0.001$, $\mathrm{OR}=28.3$ ). This means that the odds of those hospitalized for more than 24 hours were 28.3 times higher than those receiving outpatient treatments to return to the hospital within a week of discharge.

Evaluation of vital signs also showed that body temperature, respiratory rate, heart rate as well as systolic and diastolic blood pressure were significantly higher in patients who were hospitalized for more than 24 hours $(P<0.05)$ (Table 3).

\section{Discussion}

The results of our study showed no significant difference in the time of hospitalization and treatment failure between diabetic and non-diabetic patients. The strength of this study in case of diabetic wound issue is to distinguish diabetic wounds from cellulite in diabetic patients. This finding is similar to the study by Carratalà et al (10). However, the study conducted by Jenkins et al found that diabetes plays a critical role in the development of cellulite and many related diseases, including the peripheral vascular problem (11). In the study by Jenkins et al, $67 \%$ of patients had diabetes mellitus and were hospitalized longer (11). However, in the case of peripheral vascular problems, the patients with this problem in comparison to healthy patients had 14.1 and 1.23 times more chance to be hospitalized for more than 24 hours and to return within one week after treatment, respectively. These findings are consistent with the results of a study by Njim et al, which showed that peripheral vascular disease control reduces cellulite levels (12). There was a significant difference between the congenital immune deficiency, the immunocompromised, and healthy subjects at the time of hospitalization. There was also a significant difference between those who had used injectable drugs and those without a history of injections at the time of hospitalization. According to a study by Goldman et al, a combination of local eye symptoms and body temperature changes (hypo- or hyperthermia) is positively associated with increased acceptance of the emergency (13). A study by Volz et al examined the need for admission. The study concluded that patients with referred cellulitis were more likely to be hospitalized if fever, high lactate, and hand cellulitis were present (8). In a study by Sabbaj et al in 2009, it was concluded that fever is the most important criteria for hospitalization of cellulite patients referred to the emergency department (14). These results were consistent with our findings showing that body temperature, respiratory rate, heart rate, systolic and diastolic blood pressure in patients who were hospitalized for more than 24 hours were significantly higher than those who were treated in outpatient setting $(P<0.05)$. Our study showed a significant relationship between class 1 disease and hospitalization for less than 24 hours. CREST grades 2 and 3 cellulites happened more in patients hospitalized for more than 24 hours $(P<0.001)$. A study was conducted by Abiri in 2014 to determine the valid criteria for admission and discharge of patients with limb cellulitis. In their study, patients were divided according to the length of hospital stay (less than 24 hours or more than 24 hours) and CREST Guideline. The final outcome of each group was determined after one week of hospitalization. Ultimately, the treatment approach was compared to what was described in CREST Guideline. Based on our study results, the decision-making in the emergency department was very similar to CREST Guideline and almost predicted hospitalization or discharge based on what they had learned on the guideline (15). These results are consistent with our findings. Patient care is mainly intended to prevent illnesses, and outpatient setting is safer to follow-up the patients, if possible. While hospitalization is required, it will be an optimal strategy to maximize treatment of patients along with reducing the economic burden imposed on the health care system. To further achieve these goals, several studies have recommended research in this regard $(16,17)$. We recommend conducting similar researches on the relationship between the disease and the different seasons of the year. Patient management approaches should be rechecked to assess cellulite risk based on seasonal changes. Taking patients' underlying disease into account, developing new diagnostic criteria are needed to improve the patient's health regarding their special underlying disease. Towards this goal, several studies may be required in different fields of health care such as pharmacy, nursing, physiotherapy, social work and nutrition. Future research should focus on evaluating the proposed CREST score to extend its validity. Providing such privileges can help physicians set guidelines for admitting patients to the emergency department. In the present study, factors such as prescribed medication and the exact duration of hospitalization were not investigated, which was a limitation of our study due to the difficulty in follow-ups. Patients may also be referred to another treatment center for a referral after discharge.

\section{Conclusion}

The peripheral vascular problem, history of injection drug use, immunodeficiency and congenital immunodeficiency 
were significantly associated with hospitalization and treatment failure. Higher grades of CREST also had a higher length of hospitalization and a higher risk of treatment failure. Therefore, the CREST Scale is a reliable measure of the disease severity to determine the course of treatment for outpatient or inpatient treatment.

\section{Ethical issues}

Jahrom University of Medical Sciences approved the study (Approval ID: IR.JUMS.REC.1397.070).

\section{Authors Contributions}

SA, NJM, HA, and MF contributed in study design, variable selection, and revise of the study. $\mathrm{NH}$ and $\mathrm{NK}$ contributed in statistical analysis and study revise. AAA, NK, ER, and SB contributed in patient sampling, checklist design and study revise. All authors affiliated to Jahrom University of Medical Sciences contributed in patient sampling and filling the check lists in a timely manner.

\section{Acknowledgement}

We would like to thank the Clinical Research Development Unit of Peymanieh Educational and Research and Therapeutic Center of Jahrom University of Medical Sciences for providing facilities to this work.

\section{References}

1. Sukumaran V, Senanayake S. Bacterial skin and soft tissue infections. Aust Prescr 2016; 39(5): 159-63. doi: 10.18773/ austprescr.2016.058.

2. Raff AB, Kroshinsky D. Cellulitis: a review. JAMA 2016; 316(3): 325-37. doi: 10.1001/jama.2016.8825.

3. Collazos J, de la Fuente B, García A, Gómez H, Menéndez C, Enríquez H, et al. Cellulitis in adult patients: a large, multicenter, observational, prospective study of 606 episodes and analysis of the factors related to the response to treatment. PLoS One 2018; 13(9): e0204036. doi: 10.1371/ journal.pone.0204036.

4. Cranendonk DR, Lavrijsen APM, Prins JM, Wiersinga WJ. Cellulitis: current insights into pathophysiology and clinical management. Neth J Med 2017; 75(9): 366-78.

5. Sadick N. Treatment for cellulite. Int J Womens Dermatol 2019; 5(1): 68-72. doi: 10.1016/j.ijwd.2018.09.002.

6. Gabillot-Carré M, Roujeau JC. Acute bacterial skin infections and cellulitis. Curr Opin Infect Dis 2007; 20(2): 118-23. doi: 10.1097/QCO.0b013e32805dfb2d.
7. Rehder PA, EliezerET, Lane AT. Perianal cellulitis. Cutaneous group A streptococcal disease. Arch Dermatol 1988; 124(5): 702-4. doi: 10.1001/archderm.1988.01670050046018.

8. Volz KA, Canham L, Kaplan E, Sanchez LD, Shapiro NI, Grossman SA. Identifying patients with cellulitis who are likely to require inpatient admission after a stay in an ED observation unit. Am J Emerg Med 2013; 31(2): 360-4. doi: 10.1016/j.ajem.2012.09.005.

9. Ellis Simonsen SM, van Orman ER, Hatch BE, Jones SS, Gren LH, Hegmann KT, et al. Cellulitis incidence in a defined population. Epidemiol Infect 2006; 134(2): 293-9. doi: $10.1017 / s 095026880500484 x$.

10. Carratalà J, Rosón B, Fernández-Sabé N, Shaw E, del Rio $\mathrm{O}$, Rivera A, et al. Factors associated with complications and mortality in adult patients hospitalized for infectious cellulitis. Eur J Clin Microbiol Infect Dis 2003; 22(3): 151-7. doi: 10.1007/s10096-003-0902-x.

11. Jenkins TC, Knepper BC, Jason Moore S, Saveli CC, Pawlowski SW, Perlman DM, et al. Comparison of the microbiology and antibiotic treatment among diabetic and nondiabetic patients hospitalized for cellulitis or cutaneous abscess. J Hosp Med 2014; 9(12): 788-94. doi: 10.1002/ jhm.2267.

12. Njim T, Aminde LN, Agbor VN, Toukam LD, Kashaf SS, Ohuma EO. Risk factors of lower limb cellulitis in a leveltwo healthcare facility in Cameroon: a case-control study. BMC Infect Dis 2017; 17(1): 418. doi: 10.1186/s12879-0172519-1.

13. Goldman RD, Dolansky G, Rogovik AL. Predictors for admission of children with periorbital cellulitis presenting to the pediatric emergency department. Pediatr Emerg Care 2008; 24(5): 279-83. doi: 10.1097/PEC.0b013e31816ecb43.

14. Sabbaj A, Jensen B, Browning MA, Ma OJ, Newgard $\mathrm{CD}$. Soft tissue infections and emergency department disposition: predicting the need for inpatient admission. Acad Emerg Med 2009; 16(12): 1290-7. doi: 10.1111/j.15532712.2009.00536.x.

15. Abiri S. Emergency Medicine Extremity Cellulitis Referring Tothe Emergency Rooms of Hazrat Rasoul Akram and Sina Hospitals [dissertation]. Iran University of Medical Sciences; 2014.

16. Mold J. Goal-directed health care: redefining health and health care in the era of value-based care. Cureus 2017; 9(2): e1043. doi: 10.7759/cureus.1043.

17. Berwick DM, Nolan TW, Whittington J. The triple aim: care, health, and cost. Health Aff (Millwood) 2008; 27(3): 759-69. doi: 10.1377/hlthaff.27.3.759. 